\title{
ALGUNOS COLEÓPTEROS (COLEOPTERA: CARABIDAE, PROMECHEILIDAE) DE ISLAS DIEGO RAMÍREZ (563''; 6843’W), REGIÓN DE MAGALLANES
}

\author{
SOME BEETLES (COLEOPTERA: CARABIDAE, PROMECHEILIDAE) OF THE DIEGO \\ RAMIREZ ISLANDS (5632'S; 684’'W), MAGELLAN REGION
}

Mario Elgueta ${ }^{1}$, Gustavo E. Flores ${ }^{2} \&$ Sergio Roig-Juñent $^{2}$

Las islas Diego Ramírez son consideradas como sitio prioritario para la conservación, por ser un sector representativo de flora subantártica, con presencia de turbales y plantas en cojín (Manzur, 2005, Muñoz et al., 1996). Desde un punto de vista zoológico, las islas también son de gran interés, ya que constituyen un importante lugar de anidamiento de aves marinas (Vilina \& Pizarro, 2008). En cuanto a flora y fauna de este conjunto de islas, el más austral de la placa americana y el más cercano al territorio antártico, se dispone de información bastante completa sobre la biodiversidad de algas (Contreras et al., 1983), aves (Cursach et al., 2012, Schlatter \& Riveros 1997) y vegetación (Pisano, 1972, Pisano \& Schlatter 1981a, 1981b); respecto de su geología se tiene el aporte de Davidson et al., (1989).

En relación con insectos, el conocimiento de las especies presentes en islas subantárticas es bastante completo para áreas extra chilenas y la literatura al respecto es abundante (Convey \& Block, 1996, Evenhuis, 1989, Gressit 1970, Gressitt \& Pryor 1961, Gressitt \& Weber, 1959, Vogel, 1985). Para el caso de islas Diego Ramírez, en general se reconoce la presencia de insectos sólo a nivel de familias o géneros (Schlatter \& Riveros, 1997). Respecto de citas de especies, sólo se cuenta con la identificación de un ectoparásito (Palma \& Pilgrim 2002), un Noctuidae (Angulo \& Olivares 2000) y de un coleóptero (Schlatter \& Riveros, 1997) de la familia Promecheilidae, ambas especies registradas para isla Gonzalo.

En la presente Nota se entrega información sobre la identificación y antecedentes de tres especies de coleópteros (Fig. 1), en base a material recolectado por Roberto P. Schlatter en la isla Gonzalo, entre diciembre de 1980 y febrero de 1981. Se incluyen

\footnotetext{
${ }^{1}$ Area Entomología, Museo Nacional de Historia Natural, Casilla 787, Santiago (Correo central) - Chile. melgueta@mnhn.cl

2 Instituto Argentino de Investigaciones de Zonas Áridas (IADIZA), Casilla de Correo 507, 5.500 Mendoza - Argentina
} 
además otros ejemplares también recolectados en la misma isla, en febrero de 1981, por Guillermo M. Riveros. Los ejemplares recolectados se encuentran depositados en la Colección Nacional de Insectos del Museo Nacional de Historia Natural (Santiago, Chile), al igual que todo el resto del material examinado. Detalles sobre los ambientes en que se efectuaron las recolecciones se entregan en Schlatter \& Riveros (1997).

Carabidae: Migadops latus

(Guérin-Ménéville, 1841)

Migadops Waterhouse, 1842 se distribuye en el extremo sur de América, incluyendo Tierra del Fuego, e islas Malvinas (Reichardt, 1977); agrupa a sólo dos especies, ambas presentes en Chile, las que se encuentran en ambientes subantárticos (RoigJuñent y Domínguez, 2001).

Fairmaire (1885) reporta a Migadops latus para Puerto de Hambre, Tierra del Fuego, isla Navarino (Bahía Orange, Choungougon), isla Hermite, Cabo de Hornos e isla Hoste; Blackwelder (1947) agrega a su distribución a isla de los Estados e isla Madre de Dios. Lanfranco (1983) la cita para isla Deceit (Caleta Toledo), en el archipiélago de Cabo de Hornos, indicando que se presenta ahí en baja abundancia, en ambientes de bosque y turbal.

Migadops latus ha sido citada (Niemelä 1990) como la especie más abundante en la franja sur de Tierra del Fuego, en Chile y Argentina, con presencia de adultos registrada desde diciembre a febrero y mostrando preferencia por los ambientes más húmedos, en áreas cercanas al borde costero, siendo especialmente abundante en sectores aledaños a Ushuaia. No existen otros antecedentes biológicos sobre esta especie; la sinonimia se encuentra indicada en Blackwelder (1947) y Lobo (2013).

Material examinado, todo de la región de Magallanes: Puerto Williams - isla Navarino, diciembre 1963, F. Silva,4 o" o" y 1 \%; Monte Alto, 14 enero 1974, M. Martinic, 1 \%; Laguna Lynch, 28 diciembre 1968, V. Pérez, 1 o'; Lago Blanco - Tierra del Fuego, 21 marzo 1974, D. Lanfranco, 2 ơ ơ; Río Bueno - Tierra del Fuego, enero 1995, M. Elgueta, 3 o' o' y 1 \%; isla Gonzalo - Diego Ramírez, diciembre 1980 a febrero 1981, R. P. Schlatter, 1 \% . Longitud de adultos (línea media, desde mandíbulas al ápice de élitros): 8,64 - 9, $33 \mathrm{~mm}$.
Carabidae: Trechisibus (Trechisibus) antarcticus (Dejean, 1831)

Aún cuando se han propuesto subespecies (Jeannel 1962) y algunos autores las señalan explícitamente (Allegro et al., 2008, Casagranda et al., 2009, Etonti \& Mateu 1998), se ha preferido seguir el criterio de Anichtchenko (2013) y Lobo (2013); esto último se hace en consideración a que la subespecie Trechisibus antarcticus racovitzai Jeannel, 1927 se considera exclusiva de zonas boscosas de Tierra del Fuego, pero se conoce con certeza la presencia de $T$. antarcticus en diversas islas magallánicas, incluso cercanas a la anterior, y en consecuencia faltarían estudios que avalaran o confirmaran la realidad de eventuales categorías subespecíficas.

T. antarcticus se distribuye en Chile (región de Magallanes), Argentina (Tierra del Fuego, isla de los Estados) e islas Malvinas (Jeannel 1962), siendo reportada como una especie introducida en esas islas (Anichtchenko, 2013) y también en la isla Georgia del Sur, en donde se ha determinado (Brandjes et al., 1999, Ernsting et al. 1995, 1996, 1999) que este depredador afecta negativamente las poblaciones del coleóptero fitófago endémico Hydromedion sparsutum (Müller, 1884) (Promecheilidae, sensu Slipinski et al. 2010).

La introducción de esta especie en la isla Georgia del Sur se considera que ocurrió vía islas Malvinas, en donde es común, a partir de los viajes de aprovisionamiento para los asentamientos balleneros y podría ser un patrón común para explicar su presencia en otras islas con asentamiento humano; el primer registro de la presencia de esta especie en Georgia del Sur, corresponde a hallazgos cercanos a una estación ballenera abandonada (Brandjes et al., 1999).

Trechisibus Motschulsky, 1862 es un género con amplia distribución en América del Sur, aunque restringido al área andino - patagónica, que agrupa más de 100 especies y de las cuales cerca de 40 se presentan en Chile (Allegro et al., 2008, Etonti, 2003, Roig-Juñent \& Domínguez, 2001, Santamaría et al., 1991).

Material examinado, todo de la región de Magallanes: isla Hermite, 14 enero 1959, 1 ơ y 1 \%; Puerto Williams, 29 enero 1959, 3 o' o" y 1 \$; isla Gonzalo - Diego Ramírez, diciembre 1980 a 

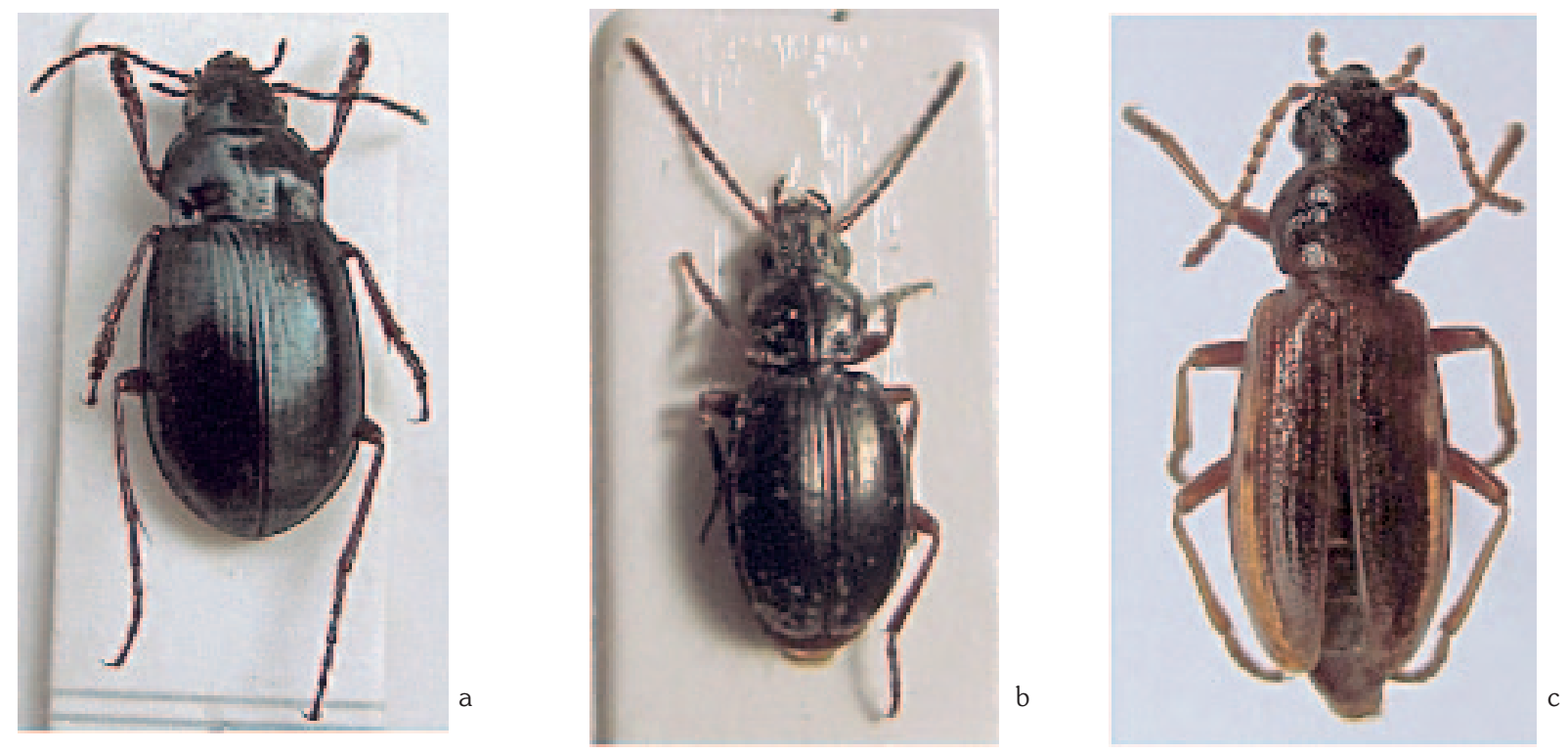

Fig. 1: Vista dorsal de adultos, machos. a) Migadops latus, longitud 8,91 mm; b) Trechisibus antarcticus, longitud 4,66 mm; c) Hydromedion anomocerum, 9,87 mm. (longitud medida en la línea media, desde mandíbulas a ápice de élitros).

febrero 1981, R. P. Schlatter, 4 o' o' y 10 \%ᄋ. Todos los ejemplares de isla Hermite y Puerto Williams se encuentran sin datos del recolector, pero con seguridad ese material debe corresponder a recolectas efectuadas por Guillermo Kuschel. Longitud de adultos (línea media, desde mandíbulas al ápice de élitros): 4,40 - 5,49 mm.

\section{Promecheilidae: Hydromedion anomocerum} Fairmaire, 1885

Las especies del género Hydromedion Waterhouse, 1875 se han reportado para Tierra del Fuego e isla Georgia del Sur, en tanto Promecheilidae es una familia de distribución austral, con representantes actuales en el extremo sur de América, isla Georgia del Sur, islas Malvinas y Tasmania (Kulzer, 1963, Flores, 1998, Slipinski et al. 2010). Aunque los antecedentes respecto de alimentación son escasos, para una especie se ha señalado que es herbívora (Ernsting et al. 1995) y lo más probable es que todas las especies sean consumidores primarios. Se presentan en ambientes abiertos en bosques de "lenga", Nohofagus pumilio (Poepp. \& Endl.) Krass. y también en ambientes de turberas y pastizales; se pueden encontrar bajo piedras en zonas abiertas, en la base de matas de hierba, bajo algas en hábitats costeros o en el fuste de lenga, bajo o en grietas de su corteza y bajo líquenes y musgos que se encuentran sobre ella.

Hydromedion anomocerum había sido citado previamente para Islote Snipe, Puerto Banner - isla Picton y Puerto Williams - isla Navarino (Kulzer, 1963, Peña, 1966); Fairmaire (1885) describe esta especie basado en material recolectado en el sector de Bahía Orange (Choungougon) en isla Navarino. De acuerdo al material de la colección del Museo Nacional de Historia Natural (Santiago, Chile) se amplía su distribución al norte del estrecho de Magallanes y para isla Gonzalo, en el archipiélago Diego Ramírez.

Material examinado, todo de la región de Magallanes: Punta Arenas, 30 enero 1969, V. Pérez, 1 \%; Rubens, 27 noviembre 1969, L. Peña G., 1 \%; Penitente, 28 febrero 1974, D. Lanfranco, 1 \%; Lago Blanco - Tierra del Fuego, 21 marzo 1974, D. Lanfranco, 1 o'; Estancia Simunovic - Porvenir, en lenga, 15 marzo 2002, C. Hernández, 1 ơ y 2 ○o; isla Gonzalo - Diego Ramírez, 12 febrero 1981, bajo piedras, M. Riveros G., 2 ơ o' y 2 \%o; isla Gonzalo - Diego Ramírez, diciembre 1980 a febrero 1981, R. P. Schlatter, 3 ơ o' y 6 ㅇ․ Longitud de 
adultos (línea media, desde mandíbulas al ápice de élitros): 7,40 - 10,29 mm.

Chanopterus Boheman, 1858 es otro género de Promecheilidae y su única especie incluida es Chanopterus paradoxus Boheman, 1858; se conoce su presencia en Tierra del Fuego (Flores 1998, Slipinski et al., 2010) y se ha citado para islas Diego Ramírez, aunque sin indicar localidad exacta (Schlatter \& Riveros, 1997). Se incluye aquí, a fin de complementar su distribución geográfica.

Material examinado, todo de la región de Magallanes: Timaukel - Tierra del Fuego, en lenga, 6 febrero 2002, C. Hernández, 1 \&; isla Gonzalo, islas Diego Ramírez, bajo algas semi secas, 20 febrero 1981, M. Riveros G., 1 \%; isla Gonzalo, islas Diego Ramírez, diciembre 1980 a febrero 1981, R. P. Schlatter, 19 . Longitud de adultos (línea media, desde mandíbulas al ápice de élitros): 6,17 - 6,58 mm.

\section{COMENTARIOS}

Desde un punto de vista biogeográfico y en consideración a las características de la vegetación presente en las islas Diego Ramírez, Pisano (1972) proponía la inclusión de esa área en una nueva provincia biótica que denominó "Gramíneo-TurbosaAntártica"; de acuerdo a análisis zoogeográfico posterior de Schlatter \& Riveros (1997), el archipiélago se incluye en la región subantártica, con proyección en la franja costera que corresponde a las islas del extremo sur de Chile.

Los insectos aquí reportados se reconocen como correspondientes a elementos de tipo subantártico. Se debe destacar que los adultos de estas especies no poseen ala metatorácica y presentan en su lugar apenas un esbozo de muñón alar. Esta imposibilidad de volar restringe notablemente su desplazamiento y, en consecuencia, sus posibilidades de dispersión. Para el caso de Trechisibus antarcticus, se ha señalado que su presencia en otras islas subantárticas obedece a transporte humano; en este caso es posible que esta misma dispersión pasiva explique su presencia en isla Gonzalo, en donde existe un refugio naval que requiere aprovisionamiento periódico. Respecto de otras especies con requerimientos ambientales más específicos, tales como aquellas de Promecheilidae, no es posible intentar una explicación de su presencia en esas islas. Es materia de futuros estudios el intentar explicar la génesis del ensamble de insectos epigeos, en el archipiélago Diego Ramírez.

\section{AGRADECIMIENTOS}

Al Profesor Roberto P. Schlatter por el depósito en el Museo Nacional de Historia Natural, de muestras de ejemplares recolectados en Isla Gonzalo.

\section{LITERATURA CITADA}

Allegro, G., Giachino, P. M. \& Sciaky, R. (2008). Notes on some Trechini (Coleoptera Carabidae) of South America with description of new species from Chile, Ecuador and Peru. Biodiversity of South America, I. Memoirs on Biodiversity, 1, 131-171. World Biodiversity Association onlus,Verona.

Angulo, A. O \& Olivares, T. S. (2000). El registro más austral de Chile de una especie de nóctuido y algunos alcances de la variación cromática (Lepidoptera: Noctuidae). Tropical Lepidoptera, 10, 69-71 (1999).

Anichtchenko, A. (2011). Trechisibus (Trechisibus) antarcticus (Dejean, 1831). En: Carabidae of the World. Sitio web: http://carabidae.org/ carabidae/taxa/antarcticus-dejean-1831.ht ml?cid=141\&img=endophallus\&max_img_ cnt $=0 \&$ main_img_cnt $=0$

Blackwelder, R. E. (1947). Checklist of the coleopterous insects of Mexico, Central America, The West Indies, and South America. United States National Museum Bulletin, 185(1), i-xii + 1-188.

Brandjes, G. J., Block, W. \& Ernsting, G. (1999). Spatial dynamics of two introduced species of carabid beetles on the sub-Antarctic island of South Georgia. Polar Biology, 21, 326-334.

Casagranda, M. D., Roig-Juñent, S. \& Szumik, C. (2009). Endemismo a diferentes escalas espaciales: un ejemplo con Carabidae (Coleoptera: Insecta) de América del Sur austral. Revista Chilena de Historia Natural, 82, 17-42.

Contreras, D., Schlatter, R. \& Ramírez, C. (1983). Flora ficológica de las islas Diego Ramírez (Chile). Serie Científica INACH, 30, 13-26.

Convey, P. \& Block, W. (1996). Antarctic Diptera: Ecology, physiology and distribution. European Journal of Entomology, 91, 1-13.

Cursach, J. A., Suazo, C. G., Schlatter, R. P. \& Rau, 
J. R. (2012). Observaciones sobre el carancho negro Phalcobaenus australis (Gmelin, 1788) en isla Gonzalo, archipiélago Diego Ramírez, Chile. Anales del Instituto de la Patagonia (Chile), 40(2), 147-150.

Davidson, J., Mpodozis, C., Godoy, E., Hervé, F. \& Muñoz, N. (1989). Jurassic accretion of a high buoyancy guyot in southernmost South America: The Diego Ramírez islands. Revista Geológica de Chile, 16(2), 247-251.

Ernsting, G., Block, W., MacAlister, H. \& Todd, C. (1995). The invasion of the carnivorous carabid beetle Trechisibus antarcticus on South Georgia (sub Antarctic) and its effect on the endemic herbivorous beetle Hydromedion sparsutum. Oecologia, 103, 3442.

Ernsting, G., Block, W. \& Brandjes, J. (1996). Trophic interactions among soil fauna in a sub-Antarctic site. Circumpolar Journal, 1-2, 56-59.

Ernsting, G., Brandjes, G. J., Block, W. \& Isaaks, J. A. (1999). Life history consequences of predation for a subantarctic beetle: evaluating the contribution of direct and indirect effects. Journal of Animal Ecology, 68, 741752.

Etonti, M. (2003). Descrizione di Trechisibus (Trechisibiodes) wardi n. sp. della Bolivia e del maschio di Trechisibus (Trechisibiodes) cyclopterus Putzeys, 1870, del Cile (Coleotera: Carabidae: Trechinae). Acta entomologica slovenica, 11(1), 129-136.

Etonti, M. \& Mateu, J. (1998). Trechini nuovi o poco noti di Argentina e Cile (Coleoptera, Carabidae, Trechinae). Bollettino del Museo Civico di Storia naturale di Venezia, 48, 65-76 (1997).

Evenhuis, N. L. 1989. Appendix I. Diptera of the Antarctic and Subantarctic Islands. Pp. 797804. In: N. L. Evenhuis (ed.), Catalog of the Diptera of the Australasian and Oceanian Regions. Honolulu: Bishop Museum Press and Leyden: E.J. Brill. Sitio web: http://hbs. bishopmuseum.org/aocat/

Fairmaire, L. (1885). Liste des coléoptères recueillis a la Terre de Feu par la Mission de la Romanche et description des espèces nouvelles. Annales de la Société Entomologique de France, 5, 33-62.

Flores, G. E. (1998). Perimylopidae. En: J. J. Morrone y S. Coscarón (eds.), Biodiversidad de artrópodos argentinos. Una perspectiva biotaxonómica (pp. 241-243.). La Plata: Ediciones Sur.

Gressitt, J. L. (1970). Subantarctic Entomology and Biogeography. Pacific Insects Monograph, 23, 295-374.

Gressitt , J. L. \& Weber, N. A. (1959). Bibliographic introduction to Antarctic and Subantarctic Entomology. Pacific Insects, 1(4), 441-480.

Gressitt, J. L. \& Pryor, M. E. (1961). Supplement to "Bibliographic introduction to Antarctic and Subantarctic Entomology". Pacific Insects, 3(4), 563-567.

Jeannel, R. (1962). Les Trechides de la Paléantarctide occidentale. En: Cl. Delamare Deboutteville \& E. Rapoport (eds.), Biologie de l'Amérique australe, 1 (pp. 527-655). Paris: Centre National de la Recherche Scientifique.

Kulzer, H. (1963). Die Helopinen des Antarktischen Gebiets (Col. Ten.). Entomologischen Arbeiten aus dem Museum G. Frey, 14, 600-629.

Lanfranco L., D. (1983). Estudios entomofaunísticos en el archipiélago del Cabo de Hornos. III. Composición y estructura de la entomofauna de suelo-superficie asociada a bosques y turbales en caleta Toledo (isla Deceit: 55⒋'S $\left.67^{\circ} 06^{\prime} \mathrm{O}\right)$. Anales del Instituto de la Patagonia (Chile), 14, 119-131.

Lobo, J. M. (2013). Proyecto Yámana - Estándar taxonómico - Coleópteros - Familia Carabidae. Sitio web: http://www.biogeografia. org/biogeografia/index.php/coleopterosfamilia-carabidae

Muñoz S., M., Núñez C., H. \& Yáñez V., J. (Editores). (1996). Libro rojo de los sitios prioritarios para la conservación de la diversidad biológica. Santiago: Corporación Nacional Forestal, Impresora Creces Ltda.

Manzur, M. I. (2005). Situación de la biodiversidad en Chile. Desafíos para la sustentabilidad. Santiago, LOM Impresores.

Niemelä, J. (1990). Habitat distribution of carabid beetles in Tierra del Fuego, South America. Entomologica Fennica, 1, 3-16.

Palma, R. L. \& Pilgrim, R. L. C. (2002). A revision of the genus Naubates (Insecta: Phthiraptera: Philopteridae). Journal of the Royal Society of New Zealand, 32(1), 7-60.

Peña G., L. E. (1966). Catálogo de los Tenebrionidae (Coleoptera) de Chile. Entomologischen Arbei- 
ten aus dem Museum G. Frey, 17, 397-453.

Pisano, E. (1972). Observaciones fito-ecológicas en las islas Diego Ramírez. Anales del Instituto de la Patagonia (Chile), 3(1-2), 161-169.

Pisano, E. \& Schlatter, R. P. (1981a). Vegetación y flora de las islas Diego Ramírez (Chile). I. Características y relaciones de la flora vascular. Anales del Instituto de la Patagonia (Chile), 12, 183-194.

Pisano, E. \& Schlatter, R. P. (1981b). Vegetación y flora de las islas Diego Ramírez (Chile). II. Comunidades vegetales vasculares. Anales del Instituto de la Patagonia (Chile), 12, 195-204.

Reichardt, H. (1977). A synopsis of the genera of Neotropical Carabidae (Insecta: Coleoptera). Quaestiones Entomologicae, 13, 346-493.

Roig-Juñent, S. \& Domínguez, M. C. (2001). Diversidad de la familia Carabidae (Coleoptera) en Chile. Revista Chilena de Historia Natural, 74, 549-571.

Santamaría, S., Balazuc, J. \& Tavares, I. I. (1991). Distribution of the European Laboulbeniales (Fungi,. Ascomycotina). An annotated list of species. Treballs de l'Institut Botanic de Barcelona, 11, 1-123.

Schlatter, R. P. \& Riveros, G. M. (1997). Historia natural del archipiélago Diego Ramírez, Chile. Serie Cientifica INACH, 47, 87-112.

Slipinski, A., Lawrence, J. F. \& Elgueta, M. (2010). 11.11. Promecheilidae Lacordaire 1859. In: Leschen, R. A. B.; Beutel, R. G.; Lawrence, J. F. (volume eds.) and A. Slipinski (Associate ed.), Handbuch der Zoologie / Handbook of Zoology, Band 4: Arthropoda, 2. Hälfte: Insecta: Coleoptera, Beetles, Teilband/Part 39, Volume 2: Morphology and Systematics (Elateroidea, Bostrichiformia, Cucujiformia partim.) (pp. 563-567) Berlin: Walter de Gruyter GmbH \& Co. KG.

Vogel, M. (1985). The distribution and ecology of epigeic invertebrates on the Subantarctic island of South Georgia. Spixiana, 8, 153-163.

Vilina, Y. \& Pizarro, C. 2008. Diversidad de especies. Aves marinas. En: CONAMA (eds.), Biodiversidad de Chile. Patrimonio y desafíos (pp. 258-265). Santiago: Ocho Libros Editores. 